\title{
Intelligent System to Support Judgmental Business Forecasting: The Case of Hotel Room Demand in Hotel Revenue Management System
}

\author{
C.Premila Rosy ${ }^{1}$, R. Ponnusamy ${ }^{2}$ \\ ${ }^{1}$ Research Scholar, Bharathiyar University, Coimbatore,Tamilnadu, India. \\ ${ }^{2}$ Principal,Rajiv Gandhi College of Engineering Nemili Tamilnadu,India.
}

\begin{abstract}
In this paper a new hotel revenue management model is proposed to provide hotel managers with a flexible and efficient decision support tool for room revenue maximization. The proposed system is composed of an advanced room demand forecast model and an optimization model that takes group reservations into account. The system also allows for expert intervention to update system parameters and outputs in order to integrate domain knowledge with computer models. A case study based on three different sets of reservation records of simulated hotel data demonstrated the viability of the system for giving useful recommendations related to accepting room requests in order to maximize hotel room revenue.
\end{abstract}

Keywords: Revenue Management System, Hotel Room Demand Forecasting, Optimal Hotel Room Allocation.

\section{Introduction}

Revenue Management (RM) is commonly practiced in the hotel industry to help hotels decide on room rate and allocation. Hotel revenue management is perceived as a managerial tool for room revenue maximization, i.e. for attempting to sell each room to the customer who is willing to pay the highest price so as to achieve the highest revenue

( El Gayar et al. 2008).

A revenue management system applies basic economic principles to pricing and controlling rooms' inventory and executes two main functions: Forecasting and optimization. The forecasting system attempts to derive future demand using historical data and current reservations activity provided by the reservations system.

With the integration of sophisticated information technology approaches and their effective combination with Machine Learning, Data Mining, Statistics, Organizational Theory and business experience and knowledge; new models of Hotel Revenue Management Systems can be developed and used to provide hotel managers with effective means to achieve an optimal level of revenue by selling hotel rooms at various price levels to different categories of customers (Shelton hotel, 2010).

Based on the demand forecast and the remaining capacity, the optimization function sets the appropriate controls on the rooms' inventory.

The use of hotel revenue management systems is reported to substantially increase revenue of hotels (The Chef hotel, 2011). On the other hand, existing RM software products are not based on integrated advanced computer methods and sophisticated prediction models, which make their results far from being comprehensive or optimal. Further development and enhancements in hotel RM models is needed and is expected to have significant impact on the tourism industry.

In this paper a new RM model is proposed to provide hotel managers with an effective tool for room revenue maximization. The proposed RM model is composed of an advanced room demand forecast model and an optimization model that addresses group reservations. The system also allows for expert intervention to update system parameters and outputs to integrate domain knowledge with computer models in order to provide a flexible and effective decision support tool for hotel revenue managers.

\section{Related Work and Problems Adressed}

Detailed and accurate forecasts are crucial to revenue management. Inaccurate predictions lead to suboptimal decisions about the rate and availability recommendations produced by the revenue management system that in turn have a negative effect on hotel revenue. In addition, accurate forecasting can also help hotels in better staffing, purchasing and budgeting decisions.

Revenue management forecasting methods fall into one of three types. Historical booking models, advanced booking models and combined models. Historical booking models only consider the final number of rooms or arrivals on a particular stay night. Advanced booking models only include the build up of reservations over time for a particular stay night. Combined models use either regression or a weighted average of historical data and advanced booking models to develop forecasts. A review of forecasting methods for all three types is 
found in Weatherford and Kimes, 2008 and Frechtling, 2001. In this study particular interest is devoted to reservation data as it is very rich and contains very useful information indicating the actual demand to come. A recent study that compares several variations of advanced booking models is found in Zakhary et al. 2011.

Generally, very little work was published on room demand forecasting. Research on revenue management forecasting is mainly based on the airline industry. Most published work on hotel revenue management systems deal with room pricing and allocation. Refer to Chiang et al., 2007 for a recent survey.

In this paper an alternative approach to room demand forecast is suggested. this approach is based on modelling hotel arrivals from its first principles and relating it with other variables/parameters like reservations, cancellations, seasonality and simulating that forward to obtain the forecast. Our proposed method is based on analyzing reservation data and extracting information- basically distributions of parameters- from past reservation to feed them in a simulator to generate future reservation.

The proposed forecasting module uses both current bookings and historical reservations and arrivals to derive accurate forecast. To the best of our knowledge, there is no published work that simulates future reservations and uses it for forecasting as the proposed system does.

In addition, modelling hotel arrivals as an interrelated process of many other factors and producing density forecast rather than point forecast, is a novel approach to hotel room demand forecasting. On the other hand, hotels offer the same rooms to different types of guests. While hotel managers would like to fill their hotels with highly profitable guests as much as possible, it is generally necessary to allow for less profitable guests in order to prevent rooms from remaining vacant.

An important decision to be made is whether to accept a booking request and generate revenue now or to reject it in anticipation of a more profitable booking request in the future (Goldman et al., 2012). Finding the right combination of guests in the hotel such that revenues are maximized is the topic of revenue management.

Revenue management is therefore defined in the hotel industry as the process of selectively accepting or rejecting customers by rate, length of stay and arrival date to maximize revenue (Vinod, 2004); by optimally matching demand to available supply (rooms) to accommodate the most profitable mix of customers.

Methods for optimal capacity utilization range from simple rule-based heuristics to sophisticated mathematical programs with hundreds of decision variables. In gold et al. briefly reviewed a subset of related mathematical programming approaches and marginal revenue approaches .

Talluri and Van Ryzin, provided a comprehensive treatment for the classic, exact and heuristic approaches to single resource capacity control. Single resource capacity control deals with optimally allocating capacity of a hotel room for a given date at different rate classes. On the other hand, the problem of managing room capacity on consecutive days when customers stay multiple nights is referred to as the network control capacity problem. This deals with a mix of customers having different lengths of stay and share the capacity on any given day. The network capacity control problem is significantly more complex than the single-resource problem and therefore its solutions rely mainly on approximations. Talluri and Van Ryzin discussed some network capacity control methods in details.

In this work group reservations are of particular interest. Group reservations - unlike individuals- are reservations filed by a larger group of people and can either be totally accepted or totally denied. Most optimization models proposed in the literature ignored group reservations and assumed a pre-defined probability distributions to represent guest arrivals (see Brumelle and Walczak, 2008 and Slyke and Young, 2010). Not taking group reservations into account is a critical limitation to the model; as group reservations can sometimes constitute the main stream for guests accommodated in the hotel, particularly in hotels targeting tourist attractions. On the other hand assuming a probability distribution for hotel arrivals can be a rather restrictive constraint in the real application of the hotel business; as hotels arrival characteristics can have a dynamically changing and evolving nature.

The RM model presented in this work treats the short comings discussed above. In particular the method presented in this paper extends existing optimization techniques for hotel revenue management to address group reservations and uses "forecasted demand" arrivals generated from the data. More details on the forecasting and the optimization models will be provided in sections 4 and 5 respectively.

\section{Overview Of The Proposed Revenue Management System}

The proposed RM model is composed of an advanced room demand forecast model and an optimization model that addresses group reservations. The system also allows for expert intervention to update system parameters and outputs in order to integrate domain knowledge with the computer model to provide a flexible and effective decision support system for revenue management.

The system performs the following functions: 1 . Scans and analyses historical booking and occupancy patterns and current reservations and fits a quantitative forecasting model (forecasting module). 2. Predictions are used as inputs to an optimization module to make rate and allocation decisions. 3. System users - which are mainly the hotel revenue managers- are allowed to change system parameters and outputs and to assess how 
these changes affect the final hotel revenue. This can help them in making better decisions regarding pricing and allocation.

The proposed forecasting module takes as input recorded reservation records from the hotel database. Each reservation record contains all the parameters that portray a certain reservation like Arrival Date, Reservation Date, Length of Stay, Room Type.... This data is then analyzed and used to extract many parameters and components like Trend, Seasonality, Booking Curve, Cancellations.... These parameters and Components are then used to generate forward reservation records that would take place in the future. The future generated reservation records are passed with all their attributes to the optimization module as an input. By analyzing these generated reservation cases one can obtain realistic perceptions for Occupancy, Arrivals, and Revenue in the future. The optimization module hence determines rates and allocations according to the estimated demand.

At any stage the revenue manager can override system outputs, like for example the forecasted demand. This might be necessary in order to include new events in the surrounding environment. After overriding system output by the revenue manager, the system is re-run based on the new changes and the reservation system is fed with the modified system output.

Figure 1 illustrates the relationship between the proposed system and the hotel environment including the existing Reservation System, Revenue Manager and the Hotel Database.

\section{Room Demand Forecast Using Simulation}

Forecasting is often considered the most critical part of revenue management. The quality of revenue Management decisions, such as pricing, capacity control, or overbooking, depends on an accurate forecast. Chiang et al. reported that a $20 \%$ reduction of forecast error can translate into a $1 \%$ incremental increase in revenue generated from the revenue management system. This section presents a new approach for room demand forecast. This approach is based on analyzing reservation data and extracting information- basically distributions of parameters- from past reservation to feed them in a simulator to generate future reservations.

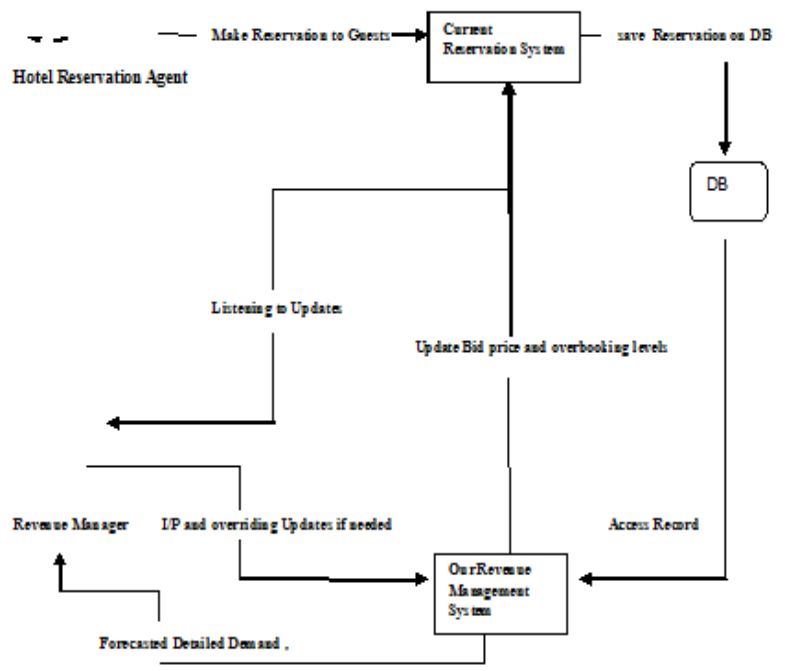

Fig1 Proposed Revenue Management System and its relationship with the Hotel Environment

Hotels store many forms of data like arrivals, reservation data, folio history, occupancy rates and more. The definitions and availability of these data may differ from one hotel to another. There are 3 forms of data that are of particular interest to this study:

1. Historical arrivals: which are the final numbers of guests that arrive in the hotel at a certain date in the past?

2. Reservation Records (RR): a detailed version of

Reservation data. A RR contains all the parameters that portray a certain reservation like (Arrival Date, Reservation Date, Length of Stay). This data should exist for all the guests that used the hotel in the past. Records must contain at least the above stated three parameters - to be useful. Other parameters like Cancellation Date, Customer Type, Room Type can be also useful.

3. Booking Matrix: a compact version of reservation data. It gives the build up of reservations for every day in the past along its booking horizon. The days in the future will have incomplete build- 
ups. Considering all the arrival dates in the past in addition to those that will take place in the future and putting them in ascending sequence will give the booking matrix.

The Reservation Records (RR) form can be considered the most important data format as the other two forms of data, stated above, can be generated from this "raw" data. Many other forms could be generated by analyzing the Reservation Records of the past.

The forecasting simulator consists of two main modules; the analysis module and the simulation module. The analysis module takes as an input the historical reservation records. It then analyzes this data and uses it to extract many parameters and components like (trend, seasonality, booking curves, cancellations dynamics and length of stay...). Certain distributions can be assumed a priori or can be set by the user; (refer to Zakhary et al, 2007). An alternative approach is to deduce the distributions and the parameter values from the data directly. For every distribution or parameter, a suitable approach is devised to estimate it. For example, the seasonal index is estimated using the multiplicative seasonal decomposition (Frechtling, 2001), the length of stay and room type distributions are estimated using a simple frequency based distribution estimator.

The effects of the average daily reservations ( $i$ days prior to arrival) and the trend and seasonality is estimated by assuming that the net reservation rate $b(i)$ equals a normalized reservation rate $b n(i)$ times a trend component $\operatorname{tr}(t)$ times a seasonal component $s(t)$, where $t$ is the arrival date, as follows:

\section{$b(i)=b n(i) * \operatorname{tr}(t) * s(t)$}

The reservation rates $b n(i)$ are estimated by grouping the reservation curves to high, medium and low according to their corresponding seasonality indexes, and estimating a template for each seasonal index.

In summary, the components extracted by the Analysis Module can be categorized into 3 classes:

- $\quad$ Easily Extracted Components: such as Booking Horizon, Arrival Time series and Cumulative Booking Matrix.

- User Defined Components: such as Forecasting Interval, and Available Rooms.

- Intelligently extracted Components: such as Seasonal Indexes, Trend, Duration of Stay Distribution, Cancellation Rate, and Booking Curve Templates.

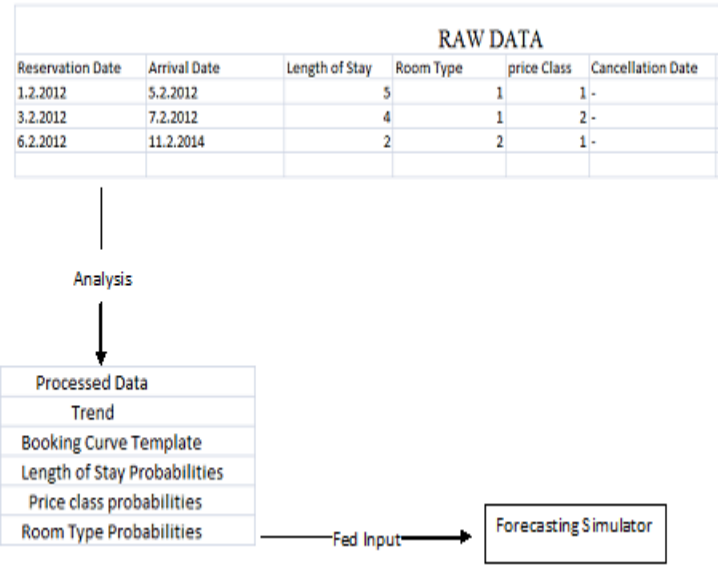

Figure 2 depicts the operation sequence of the Forecasting Simulator.

The Simulation Module takes the parameters and components estimated by the Analysis Module as an input to generate forward reservation records that would take place in the future. The future generated reservations are passed with all their attributes to the Optimization Module as an input. Analyzing these generated reservation cases; one can obtain realistic predictions for occupancy, arrivals, and revenue in the future. One advantage of this simulation approach is that one obtains distributions of future key parameters (reservations, arrivals, etc). This is performed by running the simulator many times from the current (deterministic) starting point (determined by the current snapshot of existing reservations), thereby producing many paths, each obtained by the different random components of the future reservations process. This is a 
favourable and a requested feature for the subsequent optimization module in the proposed Revenue Management System.

\section{Room Allocation Model}

The room allocation model uses the deterministic model described in literature (Baker and Collier,2003, Bertsimas et al., 2005, Goldman et al., 2002), which replaces demand by its estimation and obtains the optimal allocation of the rooms over the expected demand, i.e. it treats demand as if it was deterministic and equals it to its expectation.

The deterministic model has three dimensions to define a stay in the hotel; $(\mathrm{a}, \mathrm{L}, \mathrm{k})$, where a is the first night of the stay, $\mathrm{L}$ is the length of the stay and $\mathrm{k}$ the price class. Further, it denotes the set of stays that make use of night 1 by $\mathrm{Nl}$, where $\mathrm{Nl}=\{(\mathrm{a}, \mathrm{L}, \mathrm{k}): \mathrm{a}=1: \mathrm{a}+\mathrm{L}-1\}$. The contribution in the work described in this section is mainly adding a forth dimension to the original model to represent the size of a group $(\mathrm{g})$ in a hotel while taking into consideration the integrality constraints, which are two problems often ignored in the literature (Baker and Collier, 2003, Bertsimas et al., 2005, Goldman et al., 2002). The following are the parameters used in the model along with the final form of the modified deterministic model.

$$
\begin{aligned}
& X_{a, L, k, g}=\text { The number of roous allocated to a stay of tipe }(a, L, k, g)
\end{aligned}
$$

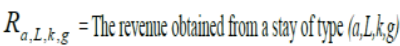

$$
\begin{aligned}
& d_{a, L, k, g}=\text { The espected demand of a stay of tipe (a,L, }, g \text { g }
\end{aligned}
$$

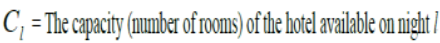

$$
\begin{aligned}
& \text { Maximize }
\end{aligned}
$$

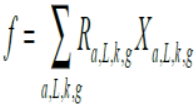

$$
\begin{aligned}
& \text { Subject to } \\
& \sum_{a, L, k, g \in V_{l}} g^{*} X_{a, L, k, g} \leq C_{l} \quad \forall \\
& X_{a, L, k, g} \leq d_{a, L, k, g} \forall a, L, k, g \\
& X_{a, L, k, g} \geq \cdot \forall a, L, k, g
\end{aligned}
$$

The major difference in this model -- beside the addition of the fourth dimension -- is the first constraint \{

$$
\sum_{a, L, k, g \in N_{l}} g_{a, L, k, g} \leq C_{l}
$$

Here the number of rooms allocated to each type of stay is multiplied by its associated group size. The objective of this model is to maximize the total revenue under the restriction that the total number of reservations for a night does not exceed the capacity of the night. Moreover, in order to prevent vacant rooms, the number of rooms allocated to each type of stay is restricted by the level of the demand, which in this model is replaced by its expectation. To incorporate stochastic demand, the randomized model found in Lai and $\mathrm{Ng}$, 2005 is used., i.e. we obtain the simulator output a number of times; and for each the modified deterministic model for groups -

Described above- is run, before and after accepting any specific request. The output for the randomized model will be the average opportunity cost. Hence a request will be only accepted if its revenue is larger than (or at least equal to) the average opportunity cost produced by the randomized model. To formally describe the idea of an opportunity-cost based control, consider a request for a stay $(a, L, k, g)$, which arrives at a point in time ' $t$ ', and its revenue is ' $r$ ' (for the case of group reservations).. This request is accepted when the following condition holds: $\mathrm{r} \geq$ fbefore - fafter

Where fbefore is the optimal total revenue generated, at time $\mathrm{t}$, by the model before accepting the request. And fafter is the optimal total revenue generated, at time t, by the model after accepting the request. The only difference between the optimization model before and after accepting the request is the capacity vector $\mathrm{Cl}$; i.e. in the model arising after a request is accepted, the capacity vector is subtracted by the amount of reserved capacity that must be allocated. 


\section{A Case Study}

This section presents the results of the proposed optimization model on a simulated hotel case study. Here a hotel manager wants to identify the optimal allocation for rooms to each type of stay a year ahead. The forecast simulator -described previously- processes reservation records that took place in the last 3 years in the hotel and extracts parameters that are used to generate forward reservation records that will take place in the coming year. From this generated data, the optimization model uses the following attributes: Arrival Date, Length of Stay, Price Class, and Group Size.

The future time horizon of the study is hence 365 nights, the maximum length a guest can stay is 7 days, and the maximum number of persons in a group is 10 persons. Moreover, the hotel manager has segmented the guests into 3 price-classes (where each class is charged differently; the first class is charged for $\$ 400$, the second for $\$ 200$ and the third is charged for $\$ 100$ ). The hotel rooms' capacity is 560 rooms. It is assumed that before the onset of the model that the initial room occupancy is $60 \%$ for the first month; and this occupancy rate decreases $10 \%$ (of total capacity) each month.

Note: Amount of price using USA Dollar is converting to Indian Rupee depending on current market value.

Based on the above information, and based on the demand scenarios generated by the simulator, the randomized model is solved as described previously. This model is categorized as a large-scale integer programming model. To solve this model a sophisticated mixed integer large-scale programming solver by JAVA /SWING is used (JDK VERSION) working under the JAVA environment. The reader may refer to (Abdel Aziz et al. 2008) for more details on the implementation.

\section{Conclusion}

This paper presents a revenue management model that is based on advanced forecasting and optimization. The proposed forecasting module attempts to model the hotel reservation process from first principles. In particular it models hotel arrivals as an interrelated process of stochastic parameters like reservations, cancellations, duration of stay, no shows, seasonality, trend, etc. In our approach we attempted to model all these processes as faithful as possible and simulate forward in time the actual process of reservations to obtain the forecast. The distinct advantages of our proposed forecasting can be summarized in the following

Points: First, it produces the density of the forecasts, and hence also confidence intervals. It also allows for scenario analysis like for example examining the effect of overbooking on future arrivals. Finally, the sensitivity of the arrival forecast due to changes of some control variables can be estimated. This, of course is a very useful feature for decision support in revenue management. Also it is worth noting that the forecasting model derives the necessary demand that the optimization model needs directly from data without the need of prior assumptions. In the future the forecasting module will also incorporate other statistical and machine learning Forecasting methods using both reservation data and historical arrivals.

On the other hand, the proposed optimization module extends existing optimization techniques for hotel revenue management to address group reservations while including integrality constraints and using "forecasted demand" arrivals generated from the data. The optimization model is based on large-scale integer programming model to optimize decision rules for accepting reservations. Results show that the model is able to generate effective recommendations to maximize revenue. For future research several more issues need to be addressed. For example, considering cancellations or guests no shows. Overbooking policies compensate for guests that would cancel or just not show up. In general, an overbooking limit should be determined in order to balance between minimizing the loss of revenue caused by empty rooms, and the loss of customers' satisfaction caused by not finding available rooms despite reserving beforehand. Also, this

paper mainly focuses on the allocation of rooms to different segments and assumes fixed prices for each segment.

\section{Acknowledgements}

We would like to thank everyone who has participated in the evaluation of the prototype system. The authors are grateful for the constructive comments of the three referees on an earlier version of this article. This research was supported in part by Hotel and Catering Management at SRM University.

\section{References}

[1]. Abdel Aziz, H. \& Saleh, M. \&El Gayar, N. \& El -Shishiny, H. (2008)A Randomized Model for Group Reservations in Hotel's Revenue Management System. Proceedings of INFOS'2008, March 27-29, pp. 24-30.

[2]. Ahmed, N. \& Atiya, A. \& El Gayar, N. \& El-Shishiny, H. (2009). An Empirical Comparison of Machine Learning Models for Time Series Forecasting", accepted for publication, to appear in Econometric Reviews.

[3]. Baker, T.K. \& Collier, D.A. (2003). The benefits of optimizing prices to manage demand in hotel revenue management systems. Production and Operations Management, Vol. 12, pp. 502-518. 
[4]. Bertsimas, D. \& de Boer, S. (2005). Simulation-based booking limits for airline revenue Management Operations Research, Vol. 53, pp.90-106.

[5]. Brumelle, S. \& Walczak, D. (2003). Dynamic airline revenue management with multiple semi-Markov demand. Operations Research. Vol. 51, pp.137-148.

[6]. Chiang, W.C \& Chen, J.C.H. \& Xu, X. (2007). An overview of research on revenue management: current issues and future research. International Journal of Revenue Management. 97-128

[7]. Choi, T.Y. \& Cho, V., (2000). Towards a knowledge discovery framework for yield management in Hong Kong hotel industry. Hospitality Management, vol. 19, p. 17-31.

[8]. Edvall, M.M. and G“oran, A.(2006). TOMLAB QUICK START GUIDE. El Gayar , N. \& Hendawi, A. M. \& Zakhary, A. \& ElShishiny, H.(2008). A Proposed Decision Support Model for Hotel Room Revenue Management. ICGST International Journal on Artificial Intelligence and Machine Learning, ICGST-AIML. Special Issue on Computational Methods for the Tourism Industry. 23-28.

[9]. Feng, Y. \& Xiao, B. (2006). Integration of pricing and capacity allocation for perishable products, European Journal of Operational Research, Vol. 168, pp.17-34.

[10]. Frechtling, D. (2001) Forecasting Tourism Demand: Methods and Strategies. Butterworth Heinemann, Oxford

[11]. Goldman, P. \& Freling, R. \& Pak, K. \& Piersma, N. (2002) Models and techniques for hotel revenue management using a rolling horizon. Journal of Revenue \& Pricing Management, Vol. 1. 207-219.

[12]. Ingold, A and McMahon-Beattie, U. and Yeoman, I. (2003). Yield Management. 2 edn, Continuum, London Lai, K-K. and Ng, WL. (2005). A stochastic approach to hotel revenue optimization. Computers \& Operations Research, Vol. 32, pp.1059-1072.

[13]. Slyke, R.V. \& Young, Y. (2000). Finite Horizon Stochastic Knapsacks with Applications to Yield Management. Journal of Operations Research. 155-172

[14]. Talluri, K.T. and van Ryzin, G.J. (2005). The theory and practice of revenue management. Springer Science + Business Media, Inc.

[15]. The MathWorks (2006) - MATLAB and Simulink for technical computing; www.mathworks.com

[16]. Vinod, B. (2004). Unlocking the value of revenue management in the hotel industry', Journal of Revenue \& Pricing Management, Vol. 3, pp.178-190.

[17]. Weatherford, L.R. and Kimes, S.E. (2006). A comparison of forecasting methods for hotel revenue management. Int. Journal of Forecasting, Vol. 19, pp.401-415.

[18]. Zakhary, A. \& Atyia A. \& El Gayar, N. \& El-Shishiny H. (2010). Forecasting Hotel Demand Using Simulation.

[19]. Technical Report. Data Mining for Improving Tourism Revenue in Egypt research project within the Egyptian Data Mining and Computer Modeling Center of Excellence.

[20]. Zakhary, A. \& El-Gayar, N \& Atyia, A. (2011). A Comparative Study of The Pickup Method and its Variations

[21]. Using A Simulated Reservation Hotel Data. ICGST International Journal on Artificial Intelligence and Machine Learning, ICGSTAIML, Special Issue on Computational Methods for the Tourism Industry. 15-21.

[22]. Zhang, D. \& Copper, W.L. (2012). Revenue management in parallel flights with customer choice Behavior. Operations Research, Vol. 53, pp.415-434.

\section{BIOGRAPHY}

Premila Rosy obtained her M.Phil degree in Computer Science from Bharathidasan University, Thiruchirappalli, in 2004. She is Currently a PhD Candidate in the Computer Science Department at Bharathiyar University, Coimbatore. She Can be Contacted at premilarosy78@gmail.com.

Ponnusamy received M.Tech. in Computer Science \& Engineering from Pondicherry University, Pondicherry, in 2000. He has also received a Ph.D. in Computer Science \& Engineering from Anna University, Chennai,in 2008. He is the Principal in the Rajiv Gandhi College of Engineering, Chennai. His areas of interests are Distributed Artificial Intelligence, Soft-Computing, E-Governance, Information Retrieval, Human-Computer Interaction and Higher-Education. He has presented/published more than 45 papers in various international conference and journals. In 2005 he got a Young Scientist Fellowship from Tamil Nadu State Council for Science \& Technology and deputed to Supercomputer Education Research Center, Indian Institute of Science, Bangalore, 2005 - 2006 as a Visiting Fellow. He can be Contacted at rponnusamy@ acm.org. 